\title{
FROM THE EDITORS' DESK
}

\section{The Primary Care Behaviorist: A New Approach to Medical/ Behavioral Integration}

\author{
Mitchell D. Feldman, MD, MPhil ${ }^{7}$ and Saul Feldman, DPA ${ }^{2}$ \\ 'Division of General Internal Medicine, Department of Medicine, University of California, San Francisco, CA, USA; ${ }^{2}$ United Behavioral Health, \\ San Francisco, CA, USA.
}

$J$ Gen Intern Med 28(3):331-2

DOI: $10.1007 / \mathrm{s} 11606-012-2330-\mathrm{z}$

(c) Society of General Internal Medicine 2013

$\mathrm{T}$ here is a growing belief in the value of integrating medical and behavioral health care, particularly for the management of depression. The Affordable Care Act endorses co-located, collaborative care, as do pilot programs funded by several foundations. Further, there is some evidence that depression is treated most effectively when primary care practitioners and behavioral health specialists collaborate. But it is not yet clear what is the best model for integration or co-location of behavioral and medical care.

In this issue of JGIM, three articles (and an accompanying editorial) examine integration and/or co-location of behavioral health services in primary care. Chang et al. ${ }^{1}$ report on the prevalence and organizational demands of three different approaches to integration of depression care in primary care practices in the Department of Veteran Affairs (VA). They found that the two models that required significant practice redesign and integration of services were less likely to be adopted than the less complex approach that favored co-location. In contrast, Szymansk et al. ${ }^{2}$ report on a VA-based retrospective cohort study of patients with positive depression screens in primary care. They conclude that in settings where mental health and primary care were integrated, patients with positive screens were more likely to receive appropriate treatment (psychotherapy or antidepressant medication) in a timely manner compared with those without integrated care. And in the column "Innovation and Improvement", Linzer et al. ${ }^{3}$ describe a novel experiment at Hennepin County Medical Center that brought together behavioral health and internal medicine providers in new collaborative arrangements to improve care for medical and psychiatric patients. These three studies offer new insights into the challenges posed by medical and behavioral health integration; however, none give us a clear roadmap of how to move forward from here.

Integration is an appealing word, conjuring up an image of social justice-integrating the public schools, for example. In behavioral health, an integrated personality is considered to be highly desirable. Yet even within the

Published online January 19, 2013 behavioral health field, there are profound and seemingly intractable integration issues that have yet to be resolved. Examples are not difficult to find. Mental health and addiction specialists often have strong ideological differences that separate them from each other and the services they provide. The gap between inpatient mental health treatment and follow-up care in the community contributes to otherwise preventable readmissions. And the mental health disciplines, trained in different silos, have little knowledge of the research published in professional journals other than their own.

The medical field is equally culpable in its failure to integrate (or even coordinate) most aspects of care. Patients discharged from the hospital are often left to their own devices to arrange appropriate and timely follow-up with their outpatient primary care practitioners (PCP). Communication and coordination of care between PCPs and subspecialists is still woefully inadequate, as it is between the various subspecialists caring for a given patient. With some notable exceptions, most health care systems deliver fragmented, poorly coordinated medical care, and as a result, often fail to meet basic quality targets, particularly for patients with multimorbidities.

The belief in and advocacy for behavioral and medical care integration has had only a modest effect on adoption rates. Integration (or co-location) remains primarily in the province of health plans. The barriers to adoption in other settings are formidable.

Chief among these barriers is the reality that while there are many advocates for medical/behavioral integration, few truly want to be integrated. PCPs and behavioral health professionals, concerned about the lack of integration within their own domains, and the inability to do much about it, may be reluctant to engage in a process that appears even more complex. Further, some health care professionals highly value their autonomy and may view integration, particularly through co-location, as a potential threat. And it is not unusual for the two professions to have what may be a distorted, but nonetheless influential view of the other - the 'medical model', for example, is anathema to many behavioral health professionals (other than psychiatrists), and some PCPs, with little training in behavioral health may not view 'talk therapy' as useful.

The slow adoption to date is evidence that despite the fact that medical and behavioral problems frequently coexist and 
should not be treated in separate silos, successful models for integration or co-location of behavioral and medical care may still be a long way off.

A new approach is needed, one that co-locates expertise rather than location. We propose the development of a new specialty within primary care, the primary care behaviorist. The primary care behaviorist (PCB) will be a primary care clinician with advanced training and certification in the diagnosis and treatment of mental and behavioral problems. PCBs are needed to help care for the great majority of patients with these problems who because of stigma, poor access to behavioral health providers and the need for treatment in the context of their other medical problems, prefer to receive treatment in the primary care setting. The PCB will also be available for consultation and comanagement with other PCPs. We expect that the PCB will, in time, receive recognition and certification similar to the "focused practice" certification now available to hospital medicine physicians through the American Board of Internal Medicine. They will be eligible for such certification only after completion of 1 year additional training and at least 3 years of clinical practice in a primary care specialty such as general internal medicine, pediatrics or family medicine.

What knowledge and skills will the PCB need? First and foremost is advanced expertise in communication and relational skills, such as active listening, use of empathy and motivational interviewing. In addition, expertise in the recognition and evidence-based treatment of behavioral and mental disorders common in primary care is essential. While the main focus would be on the common mood, anxiety and substance use disorders, PCBs will have far more expertise than their primary care colleagues with such problems as treatment resistant depression, suicidality and the initial management of bipolar disorder. The training will also include more in-depth knowledge of psychopharmacology, evidence-based psychotherapy and how to deliver brief psychotherapy (such as problem-solving therapy) in the primary care setting. The PCB must also know how behavioral health disorders interact with common medical comorbidities such as diabetes, cardiovascular disease, neurological disorders and others. And PCBs should learn enough about the behavioral health services in their communities to help patients access further behavioral health care when needed. The specific expertise required may vary somewhat, depending on the primary care specialty of the PCB; for example, pediatricians will need more expertise in attention deficit-hyperactivity disorder (ADHD), while hospital-based generalists will focus on behavioral problems common in hospitalized patients, such as delirium.

The PCB model is less complicated, more practical and far more cost-effective than integration through co-location. The goal is not to create another subspecialty (e.g. along the lines of addiction medicine) that will move expertise and patients out of the primary care setting. Rather, the PCB model more effectively integrates expertise in behavioral disorders in a single practitioner, a primary care practition$e r$, who has advanced training in the recognition and management of behavioral disorders, and who understands the challenges of practicing in the primary care setting.

Corresponding Author: Mitchell D. Feldman, MD, MPhil; Division of General Internal Medicine, Department of Medicine, University of California, San Francisco, 1545 Divisadero, San Francisco, CA 94143-0320, USA (e-mail: mfeldman@medicine.ucsf.edu).

\section{REFERENCES}

1. Chang ET, Rose DE, Yano EM, Wells KB, Metzger ME, Post EP, et al. Determinants of Readiness for Primary Care-Mental Health Integration (PC-MHI) in the VA Health Care System. J Gen Intern Med. 2013;28(3). doi: $10.1007 / \mathrm{s} 11606-012-2217-z$.

2. Szymanski BR, Bohnert KIM, Zivin K, McCarthy JF. Integrated Care: Treatment Initiation Following Positive Depression Screens. J Gen Intern Med. 2013;28(3). doi:10.1007/211606-012-2218-y.

3. Linzer M, Popkin MK, Coffey E. The Hennepin County Medical Center Program in Medical Psychiatry: Addressing the Shortened Lifespan of Patients with Mental Illness. J Gen Intern Med. 2013; doi:10.1007/s11606-012-2325-9. 